\title{
A Mismatched Filter for Integrated Sidelobe Level Minimization over a Continuous Doppler Shift Interval
}

\author{
Maria-Elisavet Chatzitheodoridi*, Abigael Taylor*, Olivier Rabaste* \\ * DEMR, ONERA, Université Paris-Saclay \\ F-91123 Palaiseau - France \\ Email: maria-elisavet.chatzitheodoridi@onera.fr, abigael.taylor@onera.fr, olivier.rabaste@onera.fr
}

\begin{abstract}
A mismatched filter that minimizes the phase code sidelobe level in the presence of Doppler shifts is presented. Taking into account the Doppler shift is essential, as for non-robust to Doppler shift phase codes, the sidelobe level may increase dramatically. The proposed filter is obtained as the solution of an optimization problem that is based on the use of the mean Integrated Sidelobe level over a given Doppler interval. Its cost function can be simply expressed as the product between the zero-Doppler cost function and the generating matrix of the Discrete Prolate Spheroidal Sequences. Comparison with known filters from the literature, for different filter lengths and Doppler interval ranges, shows that the proposed solution provides improved results in terms of mean Integrated Sidelobe Ratio over the considered Doppler interval.
\end{abstract}

Index Terms-Mismatched Filter, Optimal, Sidelobes, Doppler, Continuous, Integrated Sidelobe Ratio.

\section{INTRODUCTION}

In radar applications, target detection is usually performed via a matched filter (MF) processing that aims at maximizing the target signal-to-noise ratio (SNR) [1], [2]. This MF processing unfortunately tends to create potentially strong sidelobes in the presence of strong targets that may hide the main lobe of a weaker one, thus preventing its detection [3]. In order to mitigate potential these sidelobes, the matched filter can be replaced by a different filter, optimized with respect to the sidelobe level and not to the SNR anymore. Such a filter is denoted as a mismatched filter, since it does not use the transmitted signal for compression [3]. An extensive literature has already been devoted to the subject of mismatched filter optimization [4]-[8].

For classical linear phase steering vectors, encountered in applications such as spectral analysis or phase analysis, and chirp signals, mismatched filtering is usually performed using a weighting window [9], which provides interesting performance depending on its type, at the cost of a mainlobe enlargement. For other types of codes, mismatched filtering is usually expressed as an optimization problem defined under one or more constraints. The choice of the cost function and the associated constraints is very wide as this filtering is used in several domains such as passive radars, SAR, MIMO radars. Most papers consider the Peak-to-Sidelobe Ratio criterion and the Integrate Sidelobe Ratio criterion [6]-[8], [10], [11], mainly in the zero-Doppler case. A few papers examine the Doppler shift case [4]-[6]. Additional constraints can be also added in such optimization programs in order to control various features such as the loss-in-processing gain or the mainlobe shape [4].

In a synthetic aperture radar framework [2], [12]-[14], low sidelobes are essential so that the contrast of the generated image can be as good as possible to avoid its degradation by strong scatterers. Notably, it should be noticed that Doppler shift occurs on such situations due to the airborne radar platform motion leading to an increase of the sidelobe level. Hence the need to minimize it, by taking into consideration the intra-pulse Doppler effect. This effect is mainly observed for non-chirp signals as linear frequency modulated pulses are robust to intrapulse Doppler effect. In addition, for shared spectrum or combined radar and communication systems, chirp signals are not suitable and one may use other signals instead. In such cases, phase codes can be used, but they are generally non-robust to Doppler shifts. Notably, their sidelobes dramatically increase for high Doppler frequencies, which results in a poor image contrast. Consequently, in this article, we are interested in minimizing the Integrated Sidelobe Ratio (ISLR) of these codes over a given range of Doppler shifts, instead of the PSLR, which compares the main lobe with the higher sidelobe.

To do so, the proposed cost function is defined as the mean ISLR over a given Doppler shift interval. It can be analytically computed and expressed as a combination of the zero-Doppler cost function and the generating matrix of the Discrete Prolate Spheroidal Sequences, that facilitates its computation. This quadratic cost function is proven to be convex. Using classical constraints over the output energy and the loss-in-processing gain [4], the resulting program is quadratic convex with linear and quadratic convex constraints. It can thus be easily solved using a convex solver such as $C V X[15]$ and thus, provides a solution of the problem which is a robust optimal filter.

Performance results, obtained for two different kinds of 
phase codes and two different filter lengths, show that the proposed solution provides an improved ISLR over the considered Doppler interval.

This article is divided in 3 sections. Section II provides the mismatched filter mathematical background. More precisely, in subsection II-A, a general formulation of the mismatched filter is presented and in II-B the formulation using ISLR criterion. Section III is dedicated to the derivation of the proposed mismatched filter using a Doppler interval for intra-pulse Doppler effect. Finally, section IV presents the simulation framework and the comparative results on the proposed mismatched filter.

\section{Mathematical Formulation}

This section provides some general information about the mismatched filter formulation and the optimization criterion for the cost function definition.

\section{A. Mismatched Filter Formulation}

Let $s$ be the complex transmitted signal vector of length $N$,

$$
\mathbf{s}=\left[\begin{array}{llll}
s_{1} & s_{2} & \ldots & s_{N}
\end{array}\right]^{T}
$$

where ${ }^{T}$ is the transpose operator.

In the presence of a moving source or target, the received signal is subject to a Doppler shift $f_{d}$. Thus, the $i^{\text {th }}$ component of the received signal $\mathbf{r}$ can be expressed as:

$$
r_{i}^{f_{d}}=s_{i} e^{2 j \pi f_{d} t_{i}}, \quad \forall i \in \llbracket 1, N \rrbracket
$$

where $t_{i}=i T_{s}$, with $T_{s}$ the sampling time, such that the pulse duration is $T_{p}=N T_{s}$.

At reception, the signal is processed using a mismatched filter in order to perform target detection. The length $K$ of the mismatched filter $\mathbf{q} \in \mathbb{C}^{K}$ can be equal to or greater than the length of the signal $(K \geq N)$.

The output signal can be expressed as

$$
\mathbf{y}\left(f_{d}\right)=\mathbf{\Lambda}^{*}\left(f_{d}\right) \mathbf{q}
$$

where.$^{*}$ is the complex conjugate,

$$
\mathbf{q}=\left[\begin{array}{llll}
q_{1} & q_{2} & \ldots & q_{K}
\end{array}\right]^{T}, \quad \text { with } K \geq N
$$

and where $\boldsymbol{\Lambda}\left(f_{d}\right)$ is a correlation matrix of size $(K+N-$ 1) $\times K$, defined by

$$
\boldsymbol{\Lambda}\left(f_{d}\right)=\left[\begin{array}{ccccccc}
r_{N}^{f_{d}} & 0 & \ldots & \ldots & \ldots & \ldots & 0 \\
\vdots & r_{N}^{f_{d}} & \ddots & & & & \vdots \\
r_{2}^{f_{d}} & \vdots & \ddots & \ddots & & & \vdots \\
r_{1}^{f_{d}} & r_{2}^{f_{d}} & \ldots & r_{N}^{f_{d}} & \ddots & & \vdots \\
0 & r_{1}^{f_{d}} & \ddots & \vdots & r_{N}^{f_{d}} & \ddots & \vdots \\
\vdots & \ddots & \ddots & r_{2}^{f_{d}} & \vdots & \ddots & 0 \\
\vdots & & \ddots & r_{1}^{f_{d}} & r_{2}^{f_{d}} & \ldots & r_{N}^{f_{d}} \\
\vdots & & & \ddots & r_{1}^{f_{d}} & \ddots & \vdots \\
\vdots & & & & \ddots & \ddots & r_{2}^{f_{d}} \\
0 & \ldots & \ldots & \ldots & \ldots & 0 & r_{1}^{f_{d}}
\end{array}\right]
$$

Clearly, the classical matched filter is simply obtained by choosing $\mathbf{q}=\mathbf{s}$, when $f_{d}=0$. As already mentioned for a mismatched filter, $\mathbf{q}$ is chosen in such a way that it minimizes the sidelobe response instead of maximizing the SNR. This paper is focused on the ISLR criterion defined in the next subsection.

\section{B. Integrated Sidelobe Ratio}

Two main criteria exist in the literature when considering the sidelobe level obtained at the output of a given filter, the Peak-to-Sidelobe Ratio (PSLR) and the Integrated Sidelobe Ratio (ISLR). The latter one is defined as the ratio between the energy of all sidelobes with respect to the energy of the main lobe [16].

It is expressed as

$$
\operatorname{ISLR}(\mathbf{y})=10 \log _{10} \frac{\int_{-\infty}^{a}|\mathbf{y}(\tau)|^{2} d \tau+\int_{b}^{\infty}|\mathbf{y}(\tau)|^{2} d \tau}{\int_{a}^{b}|\mathbf{y}(\tau)|^{2} d \tau}
$$

where $\mathbf{y}$ is the output signal and the interval $[a, b]$ bounds the main lobe.

On the contrary, the PSLR criterion, widely considered in the literature [4]-[8], evaluates the difference between the main lobe level and the highest sidelobe level [16]. The motivation for choosing here the ISLR criterion flows from its great importance in SAR applications. Different scatterers may be spread all over the imaging zone, whose contributions create sidelobes that are summed up in neighbouring resolution cells. Therefore, this can not only provide potentially poor image quality but also prevent weak target detection. Besides, in such applications, having slightly higher sidelobes next to the main lobe is less inconvenient than having sidelobes that are spread along both range and azimuth directions and consequently pollute the output image.

In our framework, the ISLR criterion is thus used in order to define the cost function of the optimization prob- 
lem. Considering the sampled signal, the ISLR definition can be cast as

$$
\operatorname{ISLR}(\mathbf{y})=10 \log _{10}\left(\frac{\sum_{k \in \Omega_{S L}}\left|y_{k}\right|^{2}}{\sum_{k \in \Omega_{M L}}\left|y_{k}\right|^{2}}\right),
$$

where $\Omega_{S L}$ and $\Omega_{M L}$ respectively define the sidelobe and mainlobe sample sets. In the particular case where the sampling frequency $f_{s}=1 / T_{s}$ is chosen such that $f_{s}=B$ with $B$ the signal bandwidth, then the mainlobe set $\Omega_{M L}$ is reduced to one single sample representing the signal energy. In that case, since this signal energy is generally constrained in the mismatched filter optimization program, the ISLR criterion can be simply defined as :

$$
\operatorname{ISLR}(\mathbf{y})=10 \log _{10} \sum_{k \in \Omega_{S L}}\left|y_{k}\right|^{2} .
$$

We will use this definition in the following.

\section{Minimization Problem for Doppler-Robust Optimal Mismatched Filter}

To our knowledge, few articles in the literature have addressed the problem of optimizing the mismatched filter with respect to the sidelobe level when facing an unknown Doppler shift [4]-[6]. In these papers, the problem is generally solved in the PSLR sense and with only a small discrete set of Doppler shifts constraining the output result.

In [17], the authors propose a framework for obtaining robust filters when facing unknown parameters which take values within a given resolution cell. In the current paper, an extension of this approach is proposed in a different framework, that takes into consideration the intrapulse Doppler on a predefined interval.

\section{A. Proposed Minimization Problem}

The aim is thus to obtain the optimal filter with respect to the average ISLR over all possible Doppler shifts within a given interval.

This minimization problem can be expressed as:

$$
\begin{array}{cl}
\min _{\mathbf{q}} & \mathbb{E}_{f_{d}}\left(\left\|\mathbf{F y}\left(f_{d}\right)\right\|_{2}^{2}\right) \\
\text { s.t. } & \mathbf{s}^{H} \mathbf{q}=\mathbf{s}^{H} \mathbf{s} \\
& \mathbf{q}^{H} \mathbf{q} \leq 10^{\frac{\beta}{10}} \mathbf{s}^{H} \mathbf{s}
\end{array}
$$

where $\mathbf{F}$ is a diagonal matrix of ones except for the indices that correspond to the position of the main lobe, where the value is zero and $\beta$ is a positive constant in $\mathrm{dB}$ that expresses the loss-in-processing gain. This value represents the maximum tolerated loss for the resulting filter [4].

The first constraint allows to discard the trivial null solution and is convex because of its linearity. The second one enables to constraint the loss-in-processing gain and is also convex as it is a positive semi-definite quadratic constraint. Globally, the optimization problem is thus convex, as the 2-norm is convex, with convex constraints. As a consequence, any local optimum of the optimization problem is necessarily a global optimum.

\section{B. Computations of the Cost Function}

As mentioned, the expression (6) aims at optimizing the mismatched filter over an interval of Doppler values. Let us consider here that the Doppler frequency is uniformly distributed over the interval

$$
\Xi=\left[-\frac{\kappa}{T_{p}}, \frac{\kappa}{T_{p}}\right],
$$

with $\kappa \in \mathbb{R}_{+}^{*}$.

For example, $\kappa=\frac{1}{2}$ corresponds to the $-3 \mathrm{~dB}$ lobe width of the main lobe and $\kappa=1$ is the interval that corresponds to the full main lobe, or in other words up to the first null on each side. Note that this problem could straightforwardly be adapted to cope with non-symmetric intervals.

By injecting the compressed signal expression (2) into (6) and by noting that $\mathbf{F}^{H} \mathbf{F}=\mathbf{F}$, we obtain under the assumption of a uniform distribution of the Doppler shifts $f_{d}$ that:

$$
\begin{aligned}
\mathbb{E}_{f_{d}}\left(\left\|\mathbf{F y}\left(f_{d}\right)\right\|_{2}^{2}\right) & =\frac{T_{p}}{2 \kappa} \int_{\Xi}\left\|\mathbf{F} \mathbf{y}\left(f_{d}\right)\right\|_{2}^{2} d f_{d} \\
& \propto \mathbf{q}^{H}\left(\int_{\Xi} \boldsymbol{\Lambda}^{T}\left(f_{d}\right) \mathbf{F} \boldsymbol{\Lambda}^{*}\left(f_{d}\right) d f_{d}\right) \mathbf{q} .
\end{aligned}
$$

Let $\mathbf{M}\left(f_{d}\right)=\boldsymbol{\Lambda}^{T}\left(f_{d}\right) \mathbf{F} \boldsymbol{\Lambda}^{*}\left(f_{d}\right)$ and $\mathbf{M}_{\Xi}=\int_{\Xi} \mathbf{M}\left(f_{d}\right) d f_{d}$. Then,

$$
\begin{aligned}
\mathbb{E}_{f_{d}}\left(\left\|\mathbf{F y}\left(f_{d}\right)\right\|_{2}^{2}\right) & \propto \mathbf{q}^{H} \mathbf{M}_{\Xi} \mathbf{q} \\
& =\left\|\mathbf{M}_{\Xi}^{\frac{1}{2}} \mathbf{q}\right\|_{2}^{2} .
\end{aligned}
$$

Now, the aim is to provide an explicit expression for $\mathbf{M}_{\Xi}$. First, let us compute the matrix $\mathbf{M}(f d)$. An element of the correlation matrix $\boldsymbol{\Lambda}$ can be expressed as :

$$
\Lambda_{\mu, \nu}\left(f_{d}\right)=s_{N+\nu-\mu} e^{j 2 \pi f_{d} t_{N+\nu-\mu}},
$$

where $\mu \in \llbracket 1, K+N-1 \rrbracket$ and $\nu \in \llbracket 1, K \rrbracket$ and assuming that $s_{k}=0$ if $k \leq 0$ or $k>N$.

Using (9), the $(k, l)$ element of $\mathbf{M}$, with $k, l \in \llbracket 1, K \rrbracket$, is expressed as:

$$
M_{k, l}\left(f_{d}\right)=M_{k, l}(0) e^{\frac{2 j \pi}{N} f_{d} T_{p}(l-k)},
$$

where $\mathbf{M}(0)=\boldsymbol{\Lambda}^{H}(0) \mathbf{F} \boldsymbol{\Lambda}(0)$, which is close to the autocorrelation of the received signal for the zero-Doppler frequency and only depends on the transmitted signal s. It follows that,

$$
\begin{aligned}
\left(M_{\Xi}\right)_{k, l} & =\int_{\Xi} M_{k, l}\left(f_{d}\right) d f_{d} \\
& =M_{k, l}(0) \int_{-\frac{\kappa}{T_{p}}}^{\frac{\kappa}{T_{p}}} e^{\frac{2 j \pi}{N} f_{d} T_{p}(l-k)} d f_{d} \\
& =\frac{2 \kappa}{T_{p}} M_{k, l}(0) \operatorname{sinc}\left(\frac{2 \pi}{N} \kappa(l-k)\right) .
\end{aligned}
$$


Equation (11) provides the final expression of the $(k, l)$ entry of matrix $\mathbf{M}_{\Xi}$. It can be noted that this matrix is similar to the matrix whose eigenvectors are the Discrete Prolate Spheroidal Sequences (DPSS) [18], which are used in signal processing and communications applications. Indeed the $(k, l)$ component of the DPSS generating matrix is expressed as:

$$
P_{k, l}(\xi)=\int_{-\xi}^{\xi} e^{(2 j \pi(l-k) f)} d f=2 \xi \operatorname{sinc}(2 \pi(l-k) \xi),
$$

where $k, l \in \llbracket 1, K \rrbracket$.

Finally, the $\mathbf{M}_{\Xi}$ matrix can be written in matrix form as

$$
\mathbf{M}_{\Xi}=\frac{N}{T_{p}} \mathbf{M}(0) \odot \mathbf{P}\left(\frac{\kappa}{N}\right),
$$

where $\odot$ corresponds to the element-wise multiplication. $\mathbf{M}_{\Xi}$ thus corresponds to the element-wise multiplication of the zero-Doppler cost function and the Slepian generating matrix. It depends on the considered Doppler interval through the variable $\kappa$ and on the signal characteristics, through the pulse duration $T_{p}$, the signal length $N$ and its auto-correlation.

By injecting (12) in (8), the final form of the cost function is obtained in order to be minimized. By solving the problem (6), an optimal solution $\mathbf{q}^{*}$ can be found.

Note that if the second constraint on the loss-inprocessing gain is removed from problem (6), then the optimization problem can be analytically solved [4]. Indeed, the solution can be obtained by applying the Lagrange multipliers method and it is expressed as:

$$
\mathbf{q}_{\text {analytic }}^{*}\left(\mathbf{s}, \mathbf{M}_{\Xi}\right)=\frac{\left(\mathbf{s}^{H} \mathbf{s}\right) \mathbf{M}_{\Xi}^{-1} \mathbf{s}}{\mathbf{s}^{H} \mathbf{M}_{\Xi}^{-1} \mathbf{s}} .
$$

This analytic solution provides a sidelobe level bound for the given pulse over the whole Doppler interval in the sense that it provides the optimal mean ISLR, whatever the lossin-processing gain.

\section{Simulation and Results}

This section is dedicated to the simulation framework study and some performance results.

\section{A. Simulation Framework}

The optimal mismatched filter is coded using the Matlab toolbox CVX: Matlab Software for Disciplined Convex Programming [15]. It allows to solve efficiently the convex quadratic problem with respect to the chosen linear and quadratic constraints.

Two waveforms are considered in this paper, the Maximum Length $(M L)$ Sequence [19] and the Gold sequences [20]. Both are phase-coded waveforms and they are not as robust to Doppler shift as chirp waveforms. In particular, it has been shown that for such codes, a mismatched filter optimized considering only the zero-Doppler case may lead to a dramatic increase of the sidelobe level for non-zero Doppler shifts [4].

\begin{tabular}{||ll||}
\hline Parameter & Value \\
\hline \hline Bandwidth $B$ & $5.11 \mathrm{MHz}$ \\
Pulse duration $T_{p}$ & $10^{-4} \mathrm{~s}$ \\
Signal length $N$ & $511=\left(2^{9}-1\right)$ \\
Sampling & $\frac{1}{B}$ \\
Phase code & $\{\mathrm{ML}, \mathrm{Gold}\}$ \\
Filter length $(\# \mathbf{q})$ & $\{N, 3 N\}$ \\
Doppler interval width $\kappa$ & $\left\{\frac{1}{2}, 1,2\right\}$ \\
Allowed loss in processing gain $\beta$ & $1 \mathrm{~dB}$ \\
\hline
\end{tabular}

Table I: Parameters used for the simulations.

For both waveforms, four filters are tested and compared to each other. The classical matched filter maximizes the signal-to-noise ratio, but it is not efficient in terms of sidelobe level [1], [2] and it is denoted by ' $M F$ '. The second one is denoted by ' $M M F 0$ ', it minimizes the sidelobe level for the zero-Doppler value only and corresponds to a classical mismatched filter as proposed for instance in [4]. The third one is the proposed filter that takes into consideration any possible Doppler shifts in the interval $\Xi$ in order to reduce the sidelobes and it is denoted by ' $M M F$ '. Finally, the fourth filter corresponds to the optimal solution when the loss-in-processing gain constraint has been discarded, which is provided by (13). It is denoted by 'MMFanalytic'.

For each mismatched filter, two filter lengths are considered. The ones indicated by an index $\cdot_{N}$, correspond to the use of a filter with length equal to the signal length and the others, indicated by an index $\cdot 3 N$, correspond to a filter length equal to $3 N$.

Table I provides the values of the simulation parameters that were used for the implementation and the comparison of the results.

\section{B. Performance}

The following subsection presents illustrations of the performance obtained for different Doppler intervals and filter lengths.

Figures 1 and 2 illustrate the Integrated Sidelobe Ratio obtained for the considered filters, optimized for Gold code and ML code respectively, with respect to a given Doppler interval. Each Doppler interval depends on $\kappa$ : the smaller $\kappa$ is, the narrower the interval is going to be. Performance is provided in terms of the ISLR measured for a given Doppler shift. In Table II, the mean ISLR results over each Doppler interval are presented, which thus directly corresponds to the cost function in (6).

As expected for a given filter length, the $M M F 0$ filters provide the best ISLR performance at zero-Doppler shifts. However, the sidelobe level provided by these filters rapidly increases when increasing the Doppler shift. For this filter, it is also important to remark that the performance does not depend on the Doppler interval width, since it is optimized for the zero-Doppler shift only.

On the contrary, the $M M F$ solution provides larger sidelobes than MMFO at the neighborhood of zero-Doppler, but lower ones at higher Doppler shifts. The averaged 
ISLR on the whole considered Doppler interval is then the best, as can be seen from Table II. Here, the Doppler interval definition plays a crucial role on the performance, as it impacts the mean ISLR value obtained by the different filters.

The MMFanalytic solution presents the same behavior as the $M M F$, but generally better due to the removal of the loss-in-processing gain constraint.

As for the classical $M F$ filter, it generally provides the worst ISLR but it may provide a mean ISLR better than the $M M F 0$ solution for large Doppler intervals (see Table II).

Let us now consider the impact of the filter length. For all mismatched filter solutions, considering a longer filter, for instance in our case $3 N$ instead of $N$, provides improved performance. However the gain tends to decrease with the width of the Doppler interval. For a large interval, performance provided by both $N$ and $3 N$ lengths are almost identical and the performance provided by the MMFanalytic are also similar to those of the $M M F$. This remark combined with table III leads to the conclusion that relaxing the constraint on the loss-in-processing gain in that case will not improve the performance.

More precisely, for ML sequence, the optimal solution already verifies the second constraint of the optimization problem for any Doppler interval and filter length, as it is greater than $-1 \mathrm{~dB}$, and thus it is identical with its analytic solution, shown also in Table III. The optimal solution of the Gold pulse attains the boundary of the second constraint as the loss-in-processing gain of the analytic solution is less than $-1 \mathrm{~dB}$. However the interval length plays a crucial role, since the optimal solution tends to the analytic one while increasing it, as well as their lossin-processing gains provided in table III.

Finally, one can remark that better ISLR performance is provided by the ML sequence compared to the Gold one, which is expected since the sidelobe level of the ML code is theoretically smaller than that of the Gold one. Globally speaking, regardless of the choice of the code, the best results are provided by using the $3 N$-length $M M F$ filter as indicated in Table II.

\section{Conclusion}

The current paper proposed an optimal mismatched filter derived from a sidelobe level minimization problem for a given phase code over a specific Doppler interval. The cost function of the problem was defined with respect to the ISLR criterion. It was shown that it can be expressed as the term-by-term multiplication of the cost function in 0 and the generating matrix of the Discrete Prolate Spheroidal Sequences. The implementation results showed that, among several tested filters, the mismatched filter introduced in this paper provides in average the best energy over the considered Doppler interval, especially for long filters, as shown in table II.

\section{REFERENCES}

[1] N. Levanon and E. Mozeson, Radar signals, 3rd ed. IEEEPRESS, WILEY, 72004.

[2] B.-C. Wang, Digital signal processing techniques and applications in radar image processing. John Wiley \& Sons, 2008, vol. 91 .

[3] S. D. Blunt and E. L. Mokole, "Overview of radar waveform diversity," IEEE Aerospace and Electronic Systems Magazine, vol. 31 , no. 11 , pp. 2-42, 2016.

[4] O. Rabaste and L. Savy, "Mismatched filter optimization for radar applications using quadratically constrained quadratic programs," IEEE Transactions on Aerospace and Electronic Systems, vol. 51, no. 4, pp. 3107-3122, 2015.

[5] S. D. Blunt, K. J. Smith, and K. Gerlach, "Dopplercompensated adaptive pulse compression," in 2006 IEEE Conference on Radar. IEEE, 2006, p. 6.

[6] P. Stoica, J. Li, and M. Xue, "Transmit codes and receive filters for radar," IEEE Signal Processing Magazine, vol. 25, no. 6, pp. 94-109, 2008.

[7] J. E. Cilliers and J. C. Smit, "Pulse compression sidelobe reduction by minimization of $\mathrm{l} / \mathrm{sub} \mathrm{p} /$-norms," IEEE Transactions on Aerospace and Electronic Systems, vol. 43, no. 3, pp. 1238-1247, 2007.

[8] J. Baden and M. Cohen, "Optimal peak sidelobe filters for biphase pulse compression," in IEEE International Conference on Radar. IEEE, 1990, pp. 249-252.

[9] F. J. Harris, "On the use of windows for harmonic analysis with the discrete fourier transform," Proceedings of the IEEE, vol. 66, no. 1, pp. 51-83, Jan 1978.

[10] A. Aubry, A. De Maio, and Y. Huang, "Mimo radar beampattern design via psl/isl optimization," IEEE Transactions on Signal Processing, vol. 64, no. 15, pp. 3955-3967, Aug 2016.

[11] T. Aittomäki and V. Koivunen, "Mismatched filter design for radar waveforms by semidefinite relaxation," in 2015 IEEE International Conference on Acoustics, Speech and Signal Processing (ICASSP). IEEE, 2015, pp. 2739-2743.

[12] Y. K. Chan and V. C. Koo, "An introduction to synthetic aperture radar (sar)," Progress In Electromagnetics Research, vol. 2, pp. 27-60, 2008.

[13] I. G. Cumming and F. H. Wong, Digital Processing of Synthetic Aperture Radar Data: Algorithms and Implementation [With CDROM](Artech House Remote Sensing Library), 2005.

[14] S. W. McCandless and C. R. Jackson, "Principles of synthetic aperture radar," SAR Marine User's Manual, pp. 1-23, 2004.

[15] M. Grant and S. Boyd, "CVX: Matlab Software for Disciplined Convex Programming, version 2.1," http://cvxr.com/cvx, Mar. 2014.

[16] X. Lu and H. Sun, "Parameter assessment for sar image quality evaluation system," in 2007 st Asian and Pacific Conference on Synthetic Aperture Radar, Nov 2007, pp. 58-60.

[17] O. Rabaste and J. Bosse, "Robust mismatched filter for off-grid target," IEEE Signal Processing Letters, vol. 26, no. 8, pp. 11471151, 2019

[18] D. Slepian, "Prolate Spheroidal Wave Functions, Fourier Analysis, and Uncertainty-V: The Discrete Case," Bell System Technical Journal, vol. 57, no. 5, pp. 1371-1430, May 1978.

[19] F. J. MacWilliams and N. J. A. Sloane, "Pseudo-random sequences and arrays," Proceedings of the IEEE, vol. 64, no. 12, pp. 1715-1729, Dec 1976.

[20] R. Gold, "Optimal binary sequences for spread spectrum multiplexing (corresp.)," IEEE Transactions on Information Theory, vol. 13, no. 4, pp. 619-621, October 1967. 


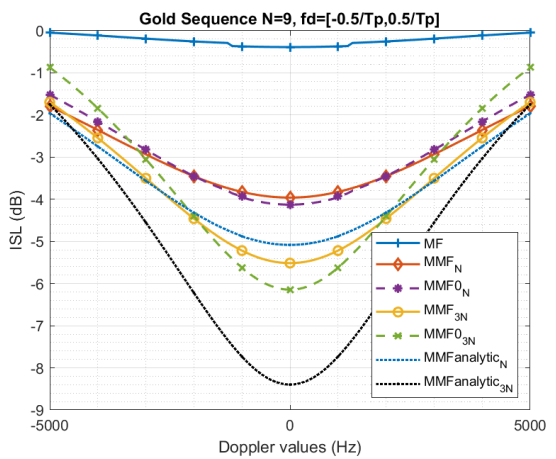

(a) $\Xi=\left[-\frac{1}{2 T_{p}}, \frac{1}{2 T_{p}}\right]$

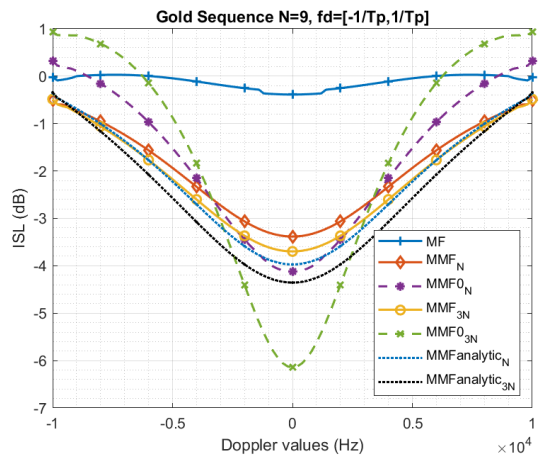

(b) $\Xi=\left[-\frac{1}{T_{p}}, \frac{1}{T_{p}}\right]$

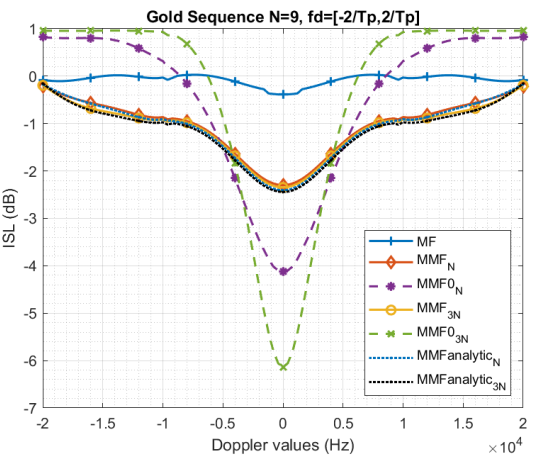

(c) $\Xi=\left[-\frac{2}{T_{p}}, \frac{2}{T_{p}}\right]$

Figure 1: Intergrated Sidelobe Ratio illustrations for Gold sequence in dB for different Doppler intervals.

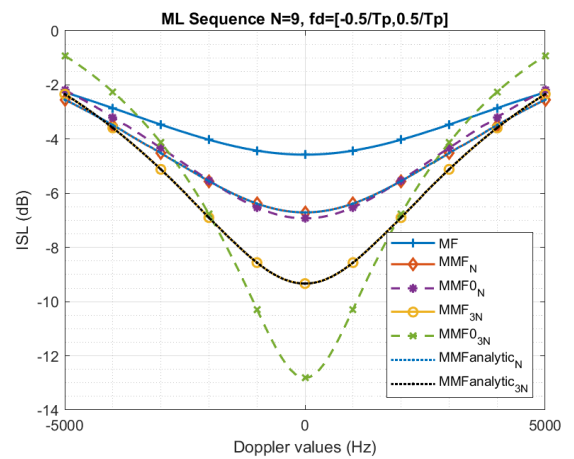

(a) $\Xi=\left[-\frac{1}{2 T_{p}}, \frac{1}{2 T_{p}}\right]$

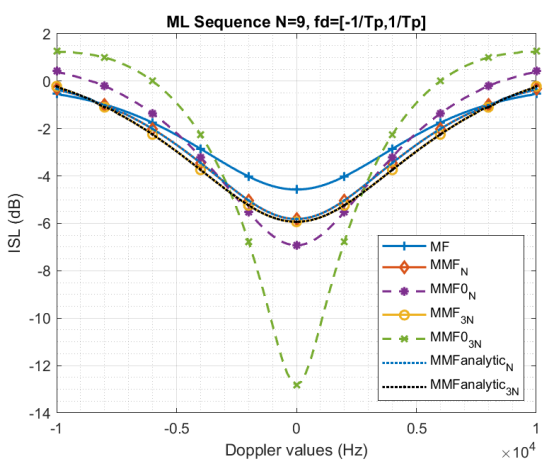

(b) $\Xi=\left[-\frac{1}{T_{p}}, \frac{1}{T_{p}}\right]$

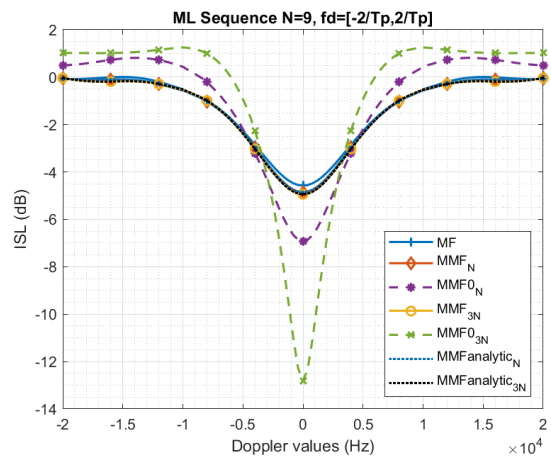

(c) $\Xi=\left[-\frac{2}{T_{p}}, \frac{2}{T_{p}}\right]$

Figure 2: Integrated Sidelobe Ratio illustrations for Maximum Length sequence in dB for different Doppler intervals.

\begin{tabular}{cccccccccc}
\hline fig & Pulse & $\kappa$ & $\mathrm{MF}$ & $\mathrm{MMF}_{N}$ & $\mathrm{MMF0}_{N}$ & $\mathrm{MMF}_{3 N}$ & $\mathrm{MMF0}_{3 N}$ & $\mathrm{MMFa}_{N}^{*}$ & $\mathrm{MMFa}_{3 N}^{*}$ \\
\hline 1a & Gold & 0.5 & -0.078 & -2.92 & -2.832 & -3.576 & -3.229 & -3.593 & -4.713 \\
1b & Gold & 1 & 0.018 & -1.768 & -1.37 & -1.951 & -0.968 & -1.996 & -2.243 \\
1c & Gold & 2 & -0.01 & -0.959 & -0.18 & -1.023 & 0.117 & -1.003 & -1.079 \\
2a & ML & 0.5 & -3.573 & -4.689 & -4.54 & -5.415 & -4.649 & -4.689 & -5.415 \\
2b & ML & 1 & -2.209 & -2.531 & -2.057 & -2.665 & -1.209 & -2.531 & -2.665 \\
2c & ML & 2 & -1.099 & -1.183 & -0.525 & -1.21 & 0.046 & -1.183 & -1.21 \\
\hline
\end{tabular}

Table II: Energy integral in dB over the whole Doppler interval defined by $\kappa$ for every filter.

* $\mathrm{MMFa}_{\{\cdot\}}$ corresponds to MMFanalytic $\{\cdot\}$

\begin{tabular}{ccccccc}
\hline fig & Pulse & $\kappa$ & $\mathrm{MMF}_{N}$ & $\mathrm{MMFa}_{N}^{*}$ & $\mathrm{MMF}_{3 N}$ & $\mathrm{MMFa}_{3 N}^{*}$ \\
\hline 1a & Gold & 0.5 & -1 & -2.894 & -1 & -3.727 \\
1b & Gold & 1 & -1 & -2.195 & -1 & -2.414 \\
1c & Gold & 2 & -1 & -1.615 & -1 & -1.692 \\
2a & ML & 0.5 & -0.391 & -0.391 & -0.564 & -0.564 \\
2b & ML & 1 & -0.194 & -0.194 & -0.274 & -0.274 \\
2c & ML & 2 & -0.069 & -0.069 & -0.092 & -0.092 \\
\hline
\end{tabular}

Table III: Loss-in-processing gain of the optimal and analytic solution in $\mathrm{dB}$ for different Doppler intervals and filter lengths.

${ }^{*} \mathrm{MMFa}_{\{\cdot\}}$ corresponds to MMFanalytic $\{\cdot\}$ 


\section{IEEE RADAR CONFERENCE, FLORENCE, ITALY \\ STUDENT PAPER CONTEST \\ SUBMISSION FORM}

The purpose of the Student Paper Competition is to recognize outstanding technical contributions from individual students.

Student Information

Student Name: $\quad$ Chatzitheodoridi Maria-Elisavet

Student Email:

maria-elisavet.chatzitheodoridi@onera.fr

University:

DEMR, ONERA, Université Paris-Saclay

Degree Program/

Expected Grad. Date:

PhD/September 2022

Advisor Name:

Abigael Taylor / Olivier Rabaste

Advisor Email:

abigael.taylor@onera.fr / olivier.rabaste@onera.fr

Student Contribution (briefly describe what the student contributed to the work):

I am a PhD student and I have done the substantial majority of the work under the supervision of my two advisors.

The motivation was given by their prior work, but I am the main contributor of this submitted paper. More precisely, I have conducted most of the theoretical and technical work under their guidance.

\section{Contest Criteria}

Please verify (by checking the box) that this submission meets the eligibility criteria:

$\square$ I (the student) am the first author, have written the paper, and have done the substantial majority of the work

$\square$ The paper contains a mature, significant contribution

$\square$ The submission consists of a clear, polished manuscript of no more than 6 pages

$\square$ If chosen as a finalist, I (the student) will register for the conference and present my work during the conference to a panel of judges. This may be in-person or remotely. The presentation will be in the same format as the lecture session presentations. 